\title{
Quantification of vascular function changes under different emotion states: A pilot study
}

\author{
Yirong Xia ${ }^{a}$, Licai Yang ${ }^{a, *}$, Xueqin Mao ${ }^{b}$, Dingchang Zheng ${ }^{c}$, Chengyu Liu ${ }^{a, *}$ \\ ${ }^{a}$ School of Control Science and Engineering, Shandong University, Jinan, 250061, China; \\ ${ }^{\mathrm{b}}$ Department of Psychology, Qilu Hospital of Shandong University, Jinan 250012, China; \\ ${ }^{c}$ Health \& Well Being Academy, Faculty of Medical Science, Anglia Ruskin University, Chelmsford, \\ CM1 1SQ, UK
}
* Author to whom correspondence should be addressed; E-Mail: yanglc@ sdu.edu.cn and bestlcy@sdu.edu.cn

\begin{abstract}
Recent studies have indicated that physiological parameters change with different emotion states. This study aimed to quantify the changes of vascular function at different emotion and sub-emotion states. Twenty young subjects were studied with their finger photoplethysmographic (PPG) pulses recorded at three distinct emotion states: natural (1 minute), happiness and sadness (10 minutes for each). Within the period of happiness and sadness emotion states, two sub-emotion states (calmness and outburst) were identified with the synchronously recorded videos. Reflection index (RI) and stiffness index (SI), two widely used indices of vascular function, were derived from the PPG pulses to quantify their differences between three emotion states, as well as between two sub-emotion states. The results showed that, when compared with the natural emotion, RI and SI decreased in both happiness and sadness emotions. The decreases in RI were significant for both happiness and sadness emotions (both $P<0.01)$, but the decreases in SI was only significant for sadness emotion $(P<0.01)$. Moreover, for comparing happiness and sadness emotions, there was significant difference in RI $(P<0.01)$, but not in SI $(P=0.9)$. In addition, significant larger RI values were observed with the outburst sub-emotion in comparison with the calmness one for both happiness and sadness emotions (both $P<0.01$ ) whereas significant larger SI values were observed with the outburst sub-emotion only in sadness emotion $(P<0.05)$. Moreover, gender factor hardly influence the RI and SI results for all three emotion measurements. This pilot study confirmed that vascular function changes with diffenrt emotion states could be quantified by the simple PPG measurement.
\end{abstract}


Keywords: Emotion recognition; PPG; Vascular function; Reflection index; Stiffness index.

\section{Introduction}

Emotion recognition has attracted increasing attention since it has a variety of useful applications in different fields. It can be applied to driver assistance system [1], to study the effects of different teaching methods [2], as well as for early diagnosis of emotion-related diseases, such as Alzheimer [3-5].

Researchers have previously studied the emotion recognition by facial expressions [6,7], speech [8] and other physiological signals, including electroencephalogram (EEG) [9], electrocardiogram (ECG) [10], skin temperature [11], photoplethysmography (PPG) and respiration signal [12]. Using physiological signal has been proven to be more effective than facial expression and speech methods as they are free from the facial masking [13]. Previous study with EEG using a fractal dimension feature showed that negative emotion had a more active response on the right hemisphere than the left one [14]. Other study with ECG has suggested that heart rate (HR) could decelerate in response to pleasant, neutral and unpleasant emotions [15]. Using temperature as an indicator, Ekman et al have demonstrated that skin temperature increased more in anger than in happiness [16]. It has also been reported that higher blood pressure (BP) was associated with decreased intensity of emotional response to both positive and negative affective contents [17].

PPG signal has also been widely used in emotion research. Different features have been extracted from PPG signals to link with different emotion states. Cheol et al reported that there was significant difference in PPG amplitude between positive and negative emotions [18]. It has also been reported that the PPG amplitude during pain and surprise emotions were significantly decreased than that during boredom emotion. Using pulse transit time (PTT), a significant decrease in surprise emotion has been reported when compared with that during boredom and pain emotions [19]. The PPI, defined as the time interval between two consecutive pulse peaks, has also been shown an accuracy of $53 \%$ for classification of sadness and happiness emotions [20]. When using the multi-features of min, max, mean and median standard deviation in pulse signals, the accuracy of $64 \%$ was achieved [21]. Moreover, pulse rate variability (PRV) has also been studied between positive and negative emotions in developing context-related computer interface devices [22].

Vascular function has been regarded as an important parameter for cardiovascular health [23,24]. 
Reflection index (RI) and stiffness index (SI) are widely used indirect measurement of vascular function [25]. However, the changes of vascular function with different emotions have not been fully investigated. This study aimed to quantify the RI and SI changes between natural, happiness and sadness emotions states. In addition, both happiness and sadness will be separated into two sub-emotion states: calmness and outburst, allowing further comparison of the RI and SI indices between the sub-emotion states. Our hypothesis is that vascular function changes with different emotion and sub-emotion states could be quantified by physiological parameters derived from simple and non-invasive PPG pulse measurement.

\section{Method}

\subsection{Subjects}

Twenty subjects with a mean age of 22 years old from Shandong University were enrolled in this study (11 females and 9 males). All subjects were the graduate students and were healthy with no history of psychotropic medication, or abuse of alcohol, confirmed by the medical examination of the University Hospital. All subjects signed the consent forms in the study. The details of subjects are summarized in Table 1.

Table 1. Basic clinical characteristics from all 20 subjects.

\begin{tabular}{cc}
\hline Variables & Value \\
\hline No. & 20 \\
Female/Male & $11 / 9$ \\
Age (year) & $22 \pm 2$ \\
Height (cm) & $169 \pm 7$ \\
Weight (kg) & $58 \pm 11$ \\
Body mass index & $20 \pm 3$ \\
\hline
\end{tabular}

Note: data are expressed as numbers or mean \pm standard deviation (SD).

\subsection{Experimental procedure}

Figure 1 shows the signal recording system (A) and the corresponding schematic diagram (B). RM6240C multi-channel signal acquirement system was used for the signal recording. A PPG sensor was attached to the left index finger of subject to record the PPG pulses. A camera was used to simultaneously record the facial expression of the subject. In order to stimulate emotion state effectively, 18 videos (9 happy ones and 9 sad ones, 10 minutes long for each) were pre-assessed and 
scored by 51 students to decide the best suitable videos for stimulating the happiness and sadness emotions. The final emotion-stimulation video for the happiness emotion is 'Who Is Undercover (a happy game)' and the one for the sadness emotion is 'Nuan Chun (a touching movie)'. The reason of employing video stimuli is that they are more natural and reliable to evoke the inner feelings of the subjects compared with other emotion stimuli such as images, sounds, etc. The previous studies also showed that the videos had a universal capacity to elicit emotions [26,27]. Figure 2 shows the still images of two emotion-stimulation videos for inducing the happiness and sadness emotions.

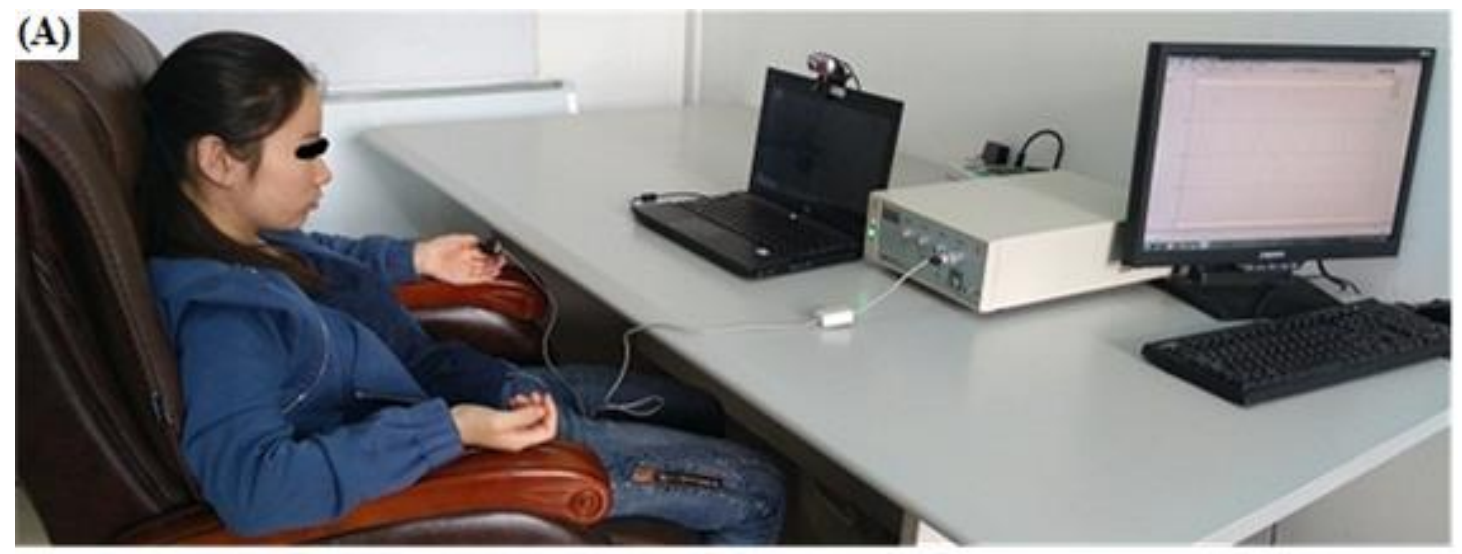

(B) Replay the emotion stimulating videos

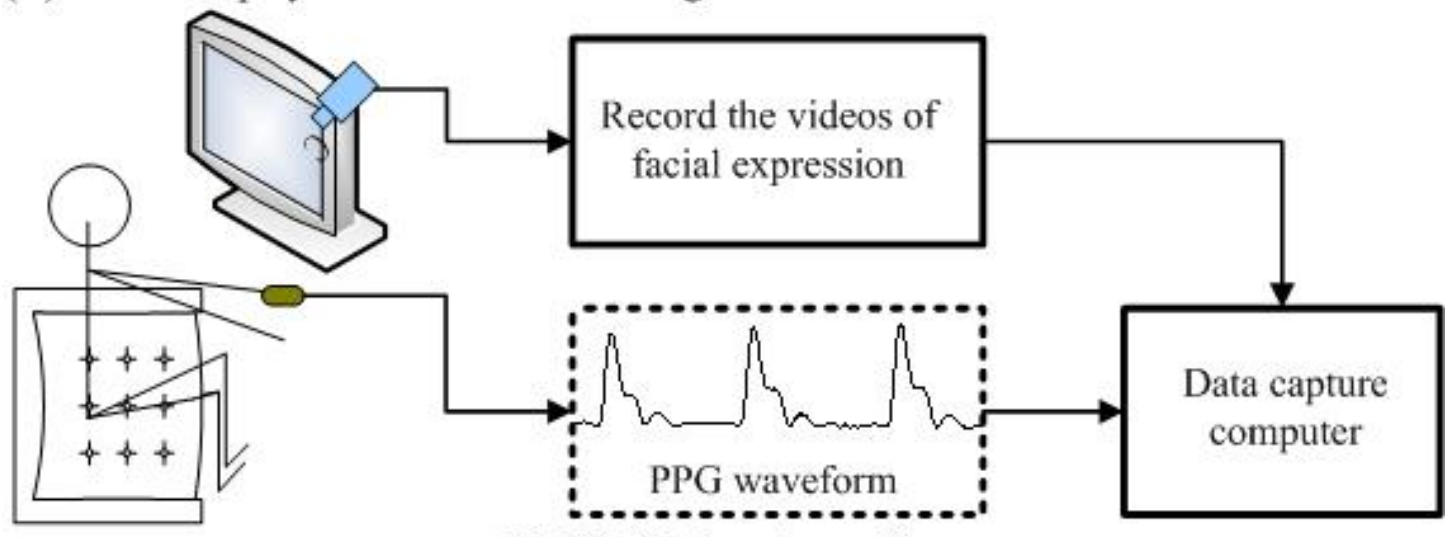

RM6240C signal recording system

Figure 1. Experimental set-up: (A) the real signal recording system and (B) the corresponding schematic diagram. 


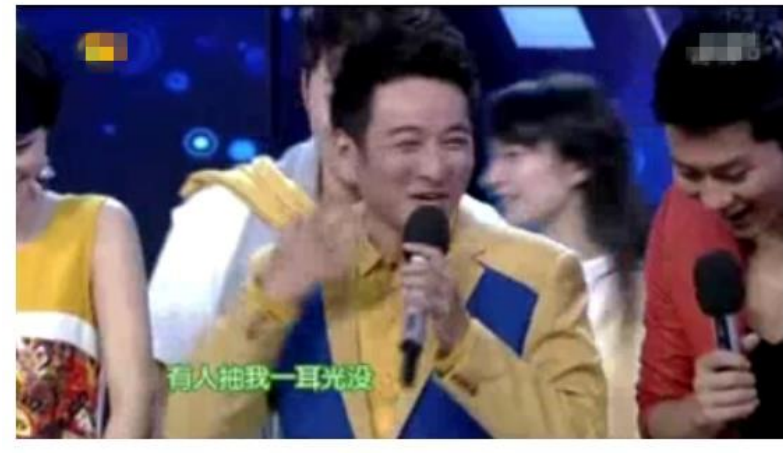

(A) Happiness

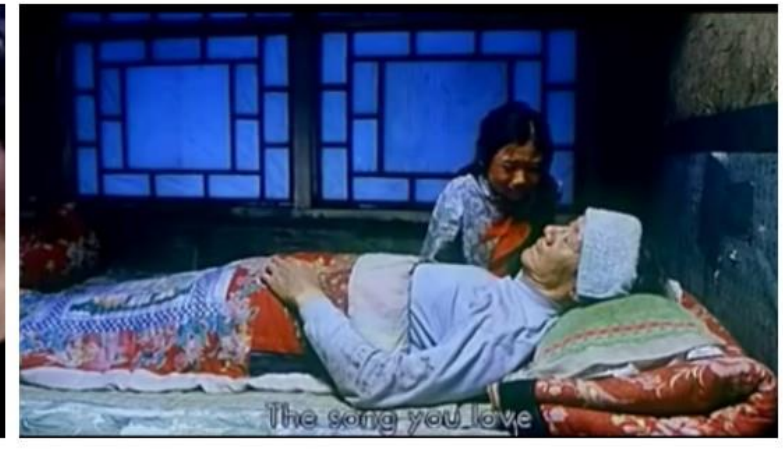

(B) Sadness

Figure 2. Still images of emotion-stimulation videos for inducing (A) happiness and (B) sadness emotions.

Before replaying the emotion-stimulation videos, 5 minutes were given to the subjects to calm them down for making a natural emotion. One minute PPG pulses were recorded during the natural emotion state. Then the emotion-stimulation videos were replayed to the subjects with randomized order. A gap of 10 minutes between the happiness and sadness emotion recordings was used to make sure the subject could restore calmness from the previous emotion state. PPG signals were recorded for 10 minutes for both happiness and sadness emotion states for each subject. All PPG signals were sampled at a rate of $800 \mathrm{~Hz}$.

\subsection{Data processing}

The Kalman filter was used [28] to remove the motion artifacts since the PPG signal is sensitive to the motion artifacts. The pulse feet were firstly detected and then the PPG signal was segmented into each single beat pulse. The pulse amplitude in each single beat was normalized $[29,30]$ and the ectopic beats were identified and excluded [31]. The mean of all normalized single beat pulses was used as the subject template for the three emotion states: natural, happiness and sadness.

Within the happiness and sadness emotion states, two sub-emotion states were manually identified by replaying the recorded facial expression videos. The two sub-emotion states were named as calmness and outburst sub-emotion states, i.e., the continuous 2 minutes with the most intense of emotion expression was identified as outburst sub-emotion and the remaining 8 minutes was identified as calmness sub-emotion state. Figure 3 shows the different emotion states and sub-emotion states with the identification of the facial expressions. The subject template at each sub-emotion state was also calculated. Figure 4 shows a PPG waveform example during the sadness emotion and the 
corresponding signal episodes during the two sub-emotion states respectively.

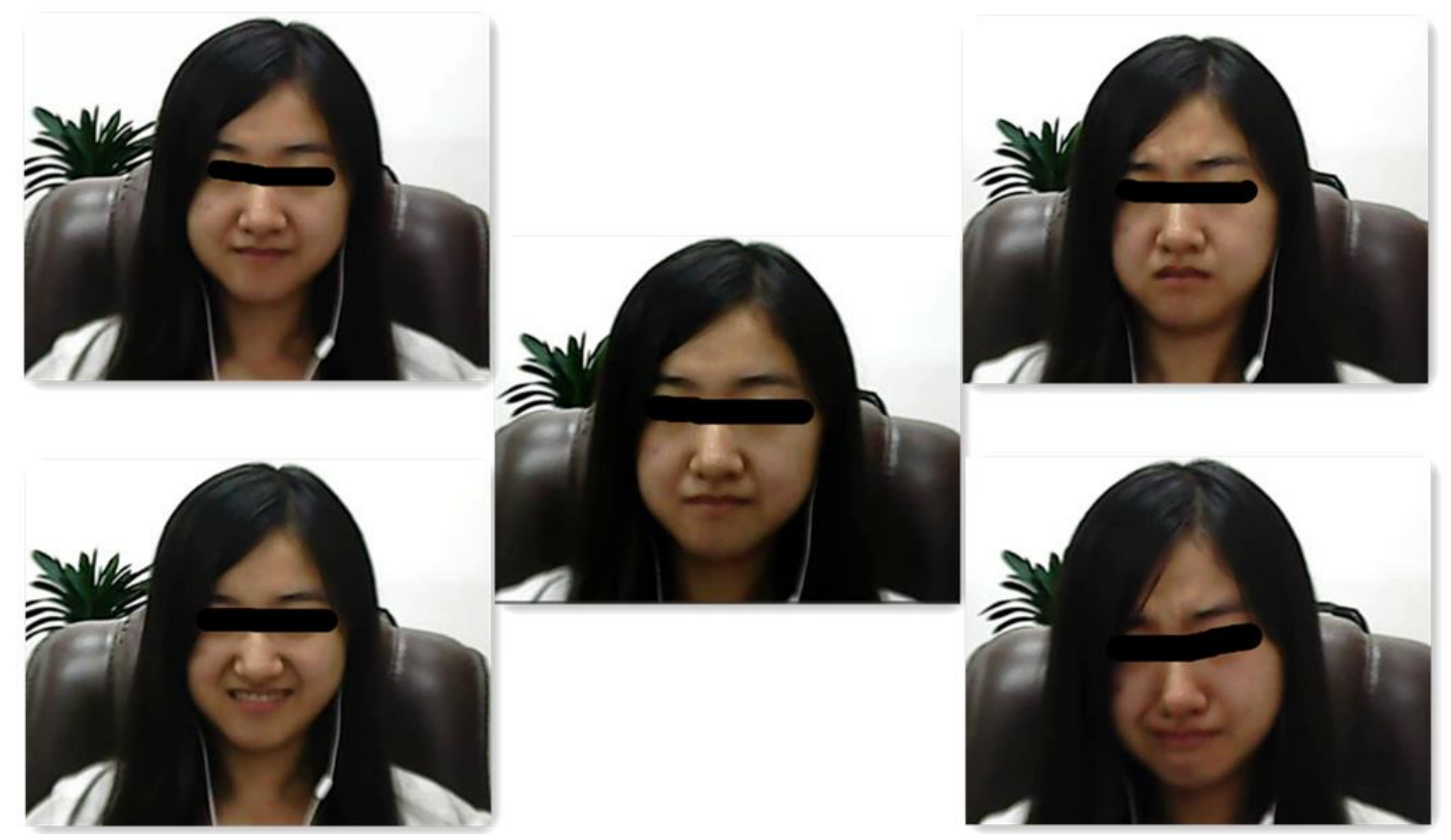

Figure 3. Different emotion states and sub-states with the identification of the facial expressions recorded by the camera: (A) calmness sub-emotion in happiness, (B) outburst sub-emotion in happiness (C) natural emotion, (D) calmness sub-emotion in sadness and (E) outburst sub-emotion in sadness.

\subsection{Definitions of reflection index and stiffness index}

Figure 5 illuminated the definitions of the indices of RI and SI on the pulse template. Index RI is the amplitude ratio between the reflected component and forward component. Index SI is the ratio of the subject height and peak to peak time (PPT) between the forward and reflected components. 

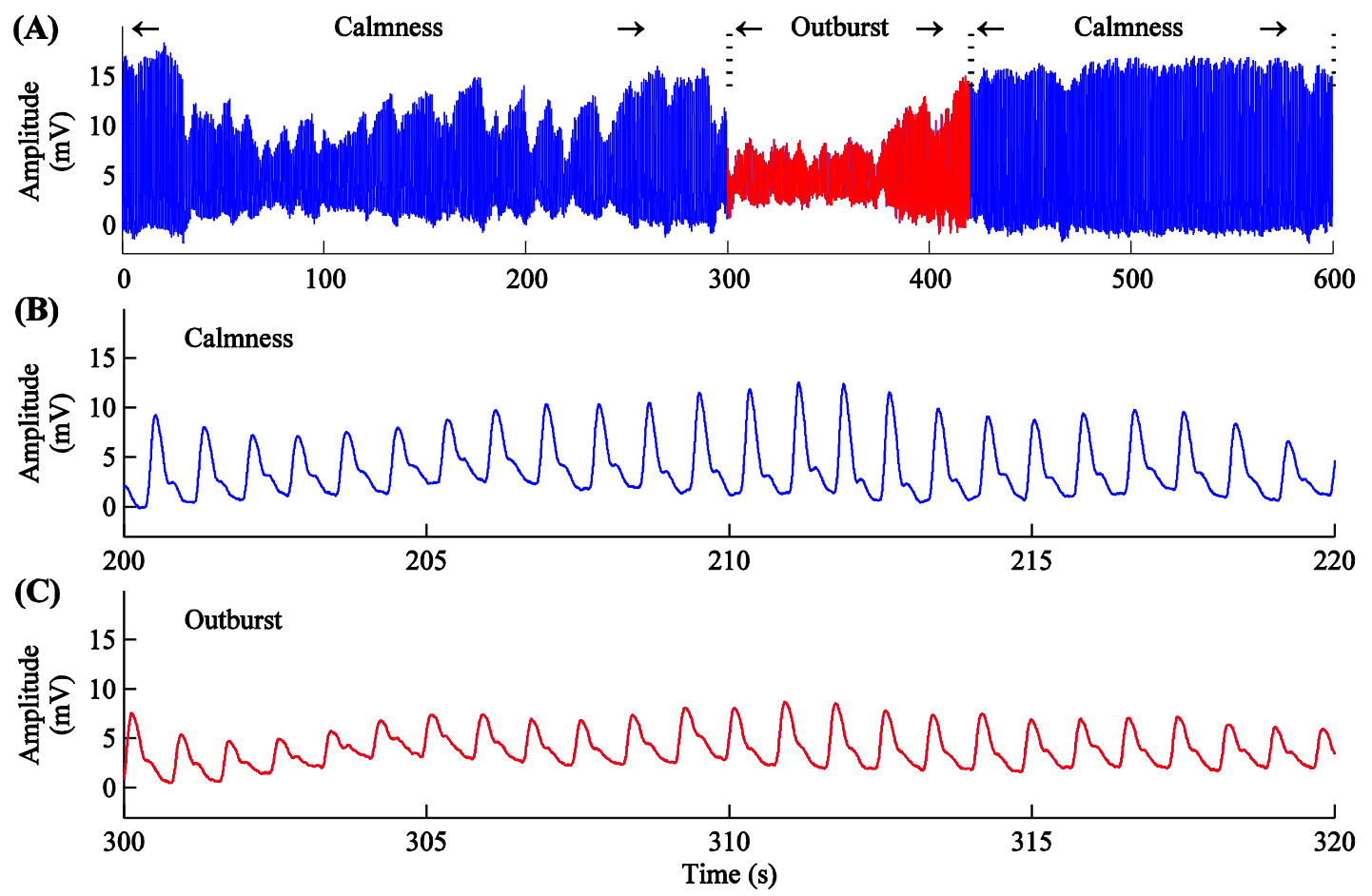

Figure 4. (A) Waveform example of the recorded PPG signal for 10 minutes during the sadness emotion and the corresponding signal episodes with 20 seconds during the two sub-emotion states: (B) calmness and $(\mathrm{C})$ outburst.

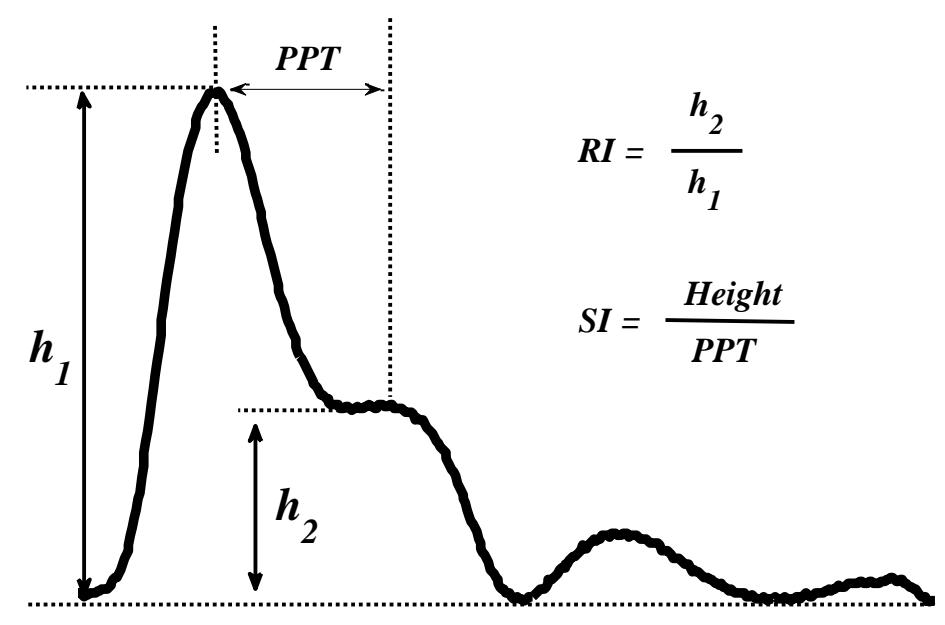

Figure 5. Definitions of the indices of RI and SI.

\subsection{Statistical analysis}

Normal distributions of the indices of RI and SI were confirmed by the Kolmogorov-Smirnov test. The RI and SI differences between the three emotion states (natural, happiness and sadness) and between the two sub-emotion states (calmness or outburst) were compared by the one-way analysis of variance (ANOVA). The effect of gender factor on the RI and SI results was also tested. All statistical 
analyses were performed using the SPSS software (Ver. 20, IBM, USA). A statistical significance was accepted at $P<0.05$.

\section{Results}

\subsection{Comparison among three emotion states}

Figure 6 shows examples of the normalized PPG pulse waveforms in single beat from the three emotion states measurements. Parameters of $P P T, h_{1}$ and $h_{2}$ defined in Figure 5 were marked. It is clear that the PPT values in both happiness and sadness emotions are smaller than that in natural emotion whereas the $h_{2}$ values are larger, resulting in both the larger RI and SI values in happiness and sadness emotions.

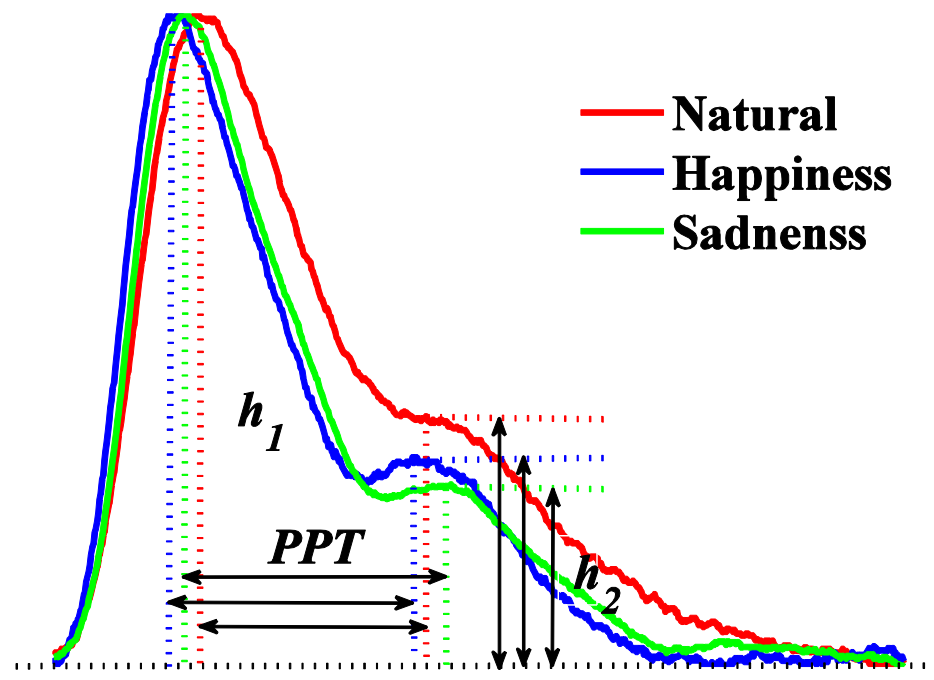

Figure 6. Examples of the normalized PPG pulse waveforms in single beat from the three emotion states measurements.

The statistical results are shown in Figure 7. In comparison with natural emotion (RI: $31.3 \pm 5.7 \%$ and SI $6.6 \pm 0.4 \mathrm{~m} / \mathrm{s}$ ), RI and SI decreased in both happiness (RI: $29.5 \pm 6.5 \%$ and SI $6.2 \pm 0.5 \mathrm{~m} / \mathrm{s}$ ) and sadness (RI: $26.8 \pm 5.4 \%$ and SI $6.2 \pm 0.4 \mathrm{~m} / \mathrm{s}$ ) emotions. For RI, the decreases were significant only in sadness emotion $(P<0.01)$, whereas for SI, it was significant in both happiness and sadness emotions (both $P<0.01)$. Moreover, RI, not SI, had significant difference $(P<0.01)$ between happiness and sadness emotion states. 

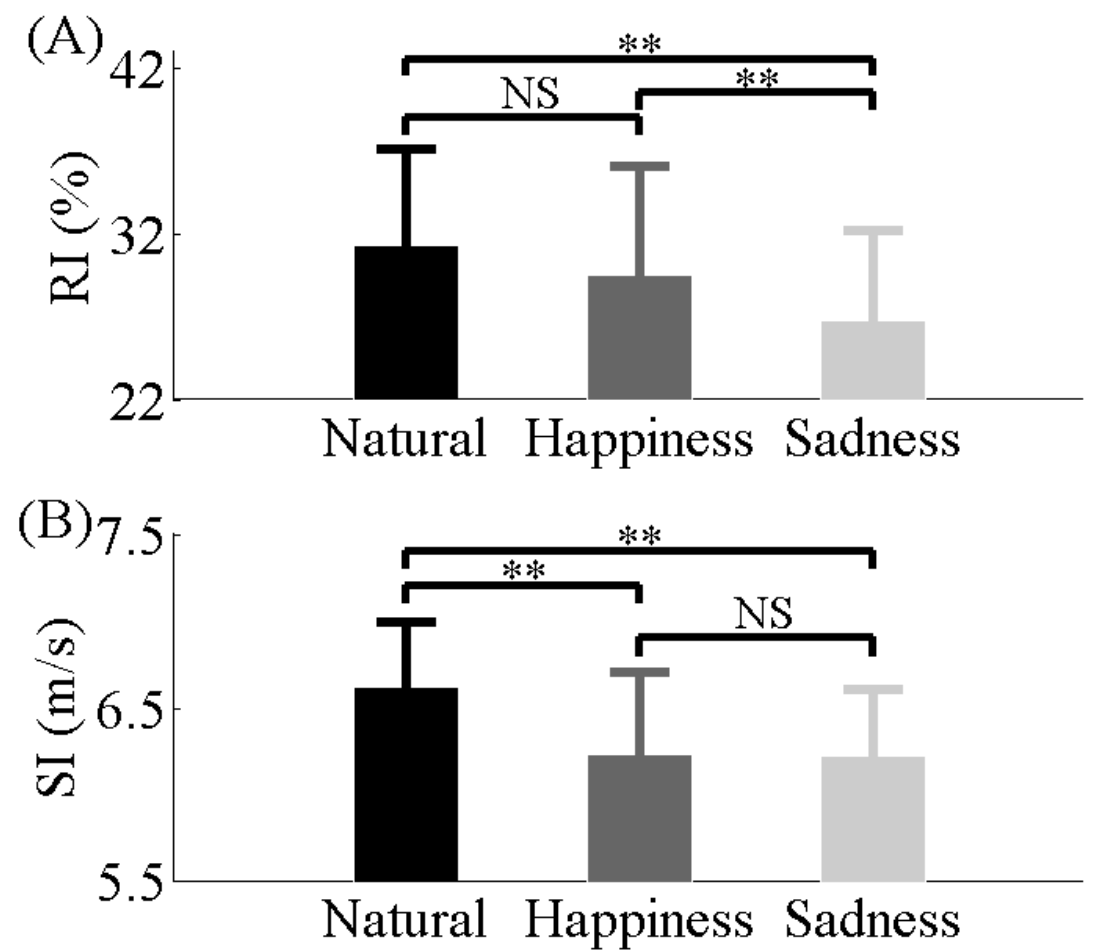

Figure 7. Mean and standard deviation (SD) of indices of RI (A) and SI (B) among the three emotion states. ** means $P<0.01$, NS means $P \geq 0.05$.

\subsection{Difference in calmness and outburst sub-emotion states}

As summarized in Table 2, for both happiness and sadness emotions, larger RI and SI values were observed with the outburst sub-emotion in comparison with the calmness sub-emotion. The increases of RI values during the outburst sub-emotion had statistical significances (both $P<0.01$ ) in both happiness and sadness emotions. However, the increase of SI values during the outburst sub-emotion had only statistical significance $(P<0.05)$ in sadness emotion.

Table 2. Results of indices of RI and SI between the two sub-emotion states.

\begin{tabular}{lcccc}
\hline \multirow{2}{*}{ Emotion } & Index & \multicolumn{2}{c}{ Sub-emotion state } & \multirow{2}{*}{ Calmness } \\
& & $28.6 \pm 5.6$ & $30.5 \pm 7.1$ & $<0.01$ \\
\hline Happiness & RI (\%) & $6.18 \pm 0.45$ & $6.26 \pm 0.51$ & 0.1 \\
& SI (m/s) & $26.0 \pm 4.5$ & $27.6 \pm 6.0$ & $<0.01$ \\
Sadness & RI (\%) & $6.16 \pm 0.31$ & $6.27 \pm 0.37$ & $<0.05$ \\
& SI (m/s) & & \\
\hline
\end{tabular}

Note: data are expressed as mean \pm standard deviation (SD). 


\subsection{Effect of gender factor on RI and SI}

Since the enrolled subjects had similar amount of females and males in this study (11 females and 9 males), the effect of gender factor on the RI and SI indices for three emotion states was tested. As shown in Table 3, group- $t$ test results showed that there was no significant difference between females and males in both RI and Si results for the three emotion states except for the SI index in sadness state, where the significant larger SI values were found in males $(6.36 \pm 0.58$ vs. $6.08 \pm 0.22 \mathrm{~m} / \mathrm{s}$ in females, $P<0.05)$.

Table 3. Effect of gender factor on the RI and SI indices for three emotion states.

\begin{tabular}{lcccc}
\hline \multirow{2}{*}{ Emotion } & Index & Female & Male & $P$-value \\
& & $31.9 \pm 5.9$ & $30.5 \pm 5.8$ & 0.6 \\
Natural & RI (\%) & $6.53 \pm 0.43$ & $6.72 \pm 0.33$ & 0.3 \\
& SI (m/s) & $29.0 \pm 6.5$ & $30.2 \pm 3.1$ & 0.6 \\
Happiness & RI (\%) & $6.07 \pm 0.51$ & $6.41 \pm 0.26$ & 0.1 \\
& SI (m/s) & $26.3 \pm 4.5$ & $27.3 \pm 3.8$ & 0.6 \\
& RI (\%) & $6.08 \pm 0.22$ & $6.36 \pm 0.58$ & $<0.05$ \\
\hline
\end{tabular}

Note: data are expressed as mean \pm standard deviation (SD).

\section{Discussion and conclusion}

Previous studies have reported the changes of different physiological features with different emotion states $[9,10,16,32]$. This polit study indicated that, vascular function changes as quantified by RI and SI from the simple PPG measurement are different among the three typically emotion states, i.e., natural, happiness and sadness emotions. This study also indicated that indices of RI and SI could be used to distinguish the two different sub-emotion states: clamness and outburst.

Studies on the emotion recognition based on PPG pulse amplitude [18], frequency and statistical characteristics $[19,20]$ were reported in previous studies. Jang and Park et al extracted pulse transit time as the feature of vascular function under three emotion states: boredom, pain and surprise, and found that the PTT index decreased under the three emotion states compared with the baseline state. In addition, the decreases in pain and surprise emotion states are statistically significant $[19,33]$. The decreases in PTT under the three emotion states (boredom, pain and surprise) suggested the decrease of the arterial compliance $[23,25]$. However, Sugawara et al studied the effect of positive emotion 
(mirthful laughter) on arterial compliance and found that laughter produces beneficial but transient effects on vascular function [34]. In the current study, we found the two widely used clinical indices: RI and SI, had lower values under happiness and sadness emotion states compared with those under natural emotion, indicating the increase of the arterial compliance. Our results are the same with Sugawara et al whereas contract with Jang and Park et al. The differences between our and other researcher results showed the different responses under different emotion states. The differences maybe also due to the different measurement positions: peripheral artery or central artery measurement. Happiness emption measurement is accompanied by the laughing action. Since the laughing action is accompanied by the contraction of thoracic, abdominal, and facial muscles, cardiac output and peripheral blood flow are expected to increase while watching a happiness stimuli (a comedy of 'Who Is Undercover' in this study). The repeated muscle contraction might have evoked increases in blood flow and resultant production of nitric oxide. Alternatively, laughing action may have evoked $\beta$-endorphin release from the pituitary gland, which activates the opiate receptors that upregulate nitric oxide synthase, as previously hypothesized [34,35]. Thus it is possible that the physical action (laughing) induced by the happiness emotion is driving the changes in vascular function. However, our study also showed different results for another emotion condition (sadness emotion) with unclear mechanism. We identify that exploring the reason of the changes in vascular function due to the different emotion states is our future work.

The significant differences of PPG features between the natural emotion and non-natural emotions also confirmed that the PPG features could carry the information of emotion state. These differences were also associated with the sympathetic activation $[16,19,32]$. Meanwhile, the larger RI and SI values observed in the outburst sub-emotion in the current study suggested the arterial stiffness increases during the more intense period of emotion expression for both happiness and sadness emotions.

One of the limitations of the current study is the relatively small number of subjects. Nevertheless, this study provided some preliminary observation on whether the widely used indices of RI and SI had statistical significance among the three distinct emotion states, and further between the two different sub-emotion states. Since the indices of RI and SI can be easily derived from PPG pulse, this study also provided the evidence that PPG could be potentially used to monitor and assess the emotion changes. 


\section{Acknowledgments}

This work was supported by the Natural Science Foundation of Shandong Province of China (2014ZRE27230) and the National Natural Science Foundation of China (61671275 and 61201049).

\section{Conflicts of Interest}

The authors declare no conflict of interest.

\section{References}

1. Eyben, F.; Wollmer, M.; Poitschke, T.; Schuller, B.; Blaschke, C.; Farber, B.; Nguyen Thien, N., Emotion on the road - necessity, acceptance, and feasibility of affective computing in the car. Advances in Human-Computer Interaction 2010, Volume 2010, ID 263593.

2. Wu, Y.W.; Wang, T.T.; Chu, X.N., Affective modeling and recognition of learning emotion: Application to e-learning. J Software 2009, 4, 859-866.

3. $\quad$ Arias Tapia, S.A.; Ratte, S.; Gomez, A.H.F.; Gonzalez Eras, A.; Barbosa, J.; Torres, J.C.; Reategui Rojas, R.; Valdiviezo Diaz, P.; Guaman Bastidas, F.; Riofrio Calderon, G.E., et al., First contribution to complex emotion recognition in patients with alzheimer's disease. In Ambient assisted living and daily activities, Pecchia, L.; Chen, L.L.; Nugent, C.; Bravo, J., Eds. Springer International Publishing: 2014; Vol. 8888, pp 341-347.

4. Wang, J.F.; Chen, B.W.; Fan, W.K.; Li, C.H., Emotion-aware assistive system for humanistic care based on the orange computing concept. Appl Comput Intell Soft Comput 2012, Volume 2012, ID 183610.

5. Tokuno, S.; Tsumatori, G.; Shono, S.; Takei, E.; Suzuki, G.; Yamamoto, T.; Mituyoshi, S.; Shimura, M. In Usage of emotion recognition in military health care: Detecting emotional change under stress, Defense Science Research Conference and Expo, Singapore, 2011; IEEE: Singapore, pp 1-5.

6. Neoha, S.C.; Zhang, L.; Mistrya, K.; Hossainb, M.A.; Limc, C.P.; Aslama, N.; Kinghorna, P., Intelligent facial emotion recognition using a layered encoding cascade optimization model. Appl Soft Comput 2015, 34, 72-93.

7. Zhang, L.; Jiang, M.; Faridc, D.; Hossaina, M.A., Intelligent facial emotion recognition and semantic-based topic detection for a humanoid robot. Expert Syst Appl 2013, 40, 5160-5168.

8. Koolagudi, S.G.; Krothapalli, S.R., Emotion recognition from speech using sub-syllabic and pitch synchronous spectral features. Int J Speech Technol 2012, 15, 495-511.

9. $\quad$ Blaiech, H.; Neji, M.; Wali, A.; Alimi, A.M. In Emotion recognition by analysis of eeg signals, 13th International Conference on Hybrid Intelligent Systems (HIS), Gammarth, 2013; IEEE: Gammarth, pp 312-318.

10. Agrafioti, F.; Hatzinakos, D.; Anderson, A.K., Ecg pattern analysis for emotion detection. IEEE Trans Affect Comput 2012, 3, 102-115.

11. Kim, D.; Ahn, S.; Park, S.; Whang, M., Interactive emotional lighting system using physiological signals. IEEE Trans Consumer Electron 2013, 59, 765-771.

12. Wu, C.K.; Chung, P.C.; Wang, C.J., Representative segment-based emotion analysis and classification with automatic respiration signal segmentation. IEEE Trans Affect Comput 2012, 3, 482-495.

13. Van den Broek, E.L.; Lisy, V.; Janssen, J.H.; Westerink, J.H.; Schut, M.H.; Tuinenbreijer, K., Affective man-machine interface: Unveiling human emotions through biosignals. In Biomedical engineering 
systems and technologies, Fred, A.; Filipe, J.; Gamboa, H., Eds. Springer Berlin Heidelberg: 2010; Vol. 52, pp 21-47.

14. Liu, Y.S.; Sourina, O. In Eeg databases for emotion recognition, 2013 International Conference on Cyberworlds, Yokohama, 2013; IEEE: Yokohama, pp 302-309.

15. Abercrombie, H.C.; Chambers, A.S.; Greischar, L.; Monticelli, R.M., Orienting, emotion, and memory: Phasic and tonic variation in heart rate predicts memory for emotional pictures in men. Neurobiol. Learn. Mem. 2008, 90, 644-650.

16. Ekman, P.; Levenson, R.W.; Friesen, W.V., Autonomic nervous system activity distinguishes among emotions. Science 1983, 221, 1208-1210.

17. McCubbin, J.A.; Merritt, M.M.; Sollers, J.J.; Evans, M.K.; Zonderman, A.B.; Lane, R.D.; Thayer, J.F., Cardiovascular-emotional dampening: The relationship between blood pressure and recognition of emotion. Psychosom Med 2011, 73, 743-750.

18. Cheol, W.M.; Woo, J., The study on emotion recognition by time-dependent parameters of autonomic nervous response. Kr J Sci Emot Sen 2008, 11, 637-644.

19. Jang, E.H.; Park, B.J.; Park, M.S.; Kim, S.H.; Sohn, J.H., Analysis of physiological signals for recognition of boredom, pain, and surprise emotions. J Physiol Anthropol 2015, 34, 25.

20. Park, M.W.; Kim, C.J.; Whang, M.; Lee, E.C. In Individual emotion classification between happiness and sadness by analyzing photoplethysmography and skin temperature, Fourth World Congress onSoftware Engineering, Hongkong, Dec., 2013; IEEE: Hongkong, pp 190-194.

21. Zhang, H.L.; Liu, G.Y. In Research of emotion recognition based on pulse signal, Third International Conference on Advanced Computer Theory and Engineering, Chengdu, 2010; IEEE: Chengdu, pp 506-509.

22. Yoo, S.K.; Lee, C.; Lee, G.; Lee, B.; Jeong, K.; Park, Y., Portable device for bi-emotional state identification using heart rate variability. In Ubiquitous computing systems, Youn, H.Y.; Kim, M.; Morikawa, H., Eds. Springer Berlin Heidelberg: 2006; Vol. 4239, pp 528-536.

23. Liu, C.Y.; Zheng, D.C.; Zhao, L.N.; Li, P.; Li, B.; Murray, A.; Liu, C.C., Elastic properties of peripheral arteries in heart failure patients in comparison with normal subjects. J Physiol Sci 2013, 63, 195-201.

24. Liu, C.Y.; Zheng, D.C.; Zhao, L.N.; Liu, C.C., Gaussian fitting for carotid and radial artery pressure waveforms: Comparison between normal subjects and heart failure patients. Biomed Mater Eng 2014, 24, 271-277.

25. Millasseau, S.C.; Ritter, J.M.; Takazawa, K.; Chowienczyk, P.J., Contour analysis of the photoplethysmographic pulse measured at the finger. J Hypertens 2006, 24, 1449-1456.

26. Sato, W.; Noguchi, M.; Yoshikawa, S., Emotion elicitation effect of films in a japanese sample. Social Behavior and Personality 2007, 35, 863-874.

27. Baumgartner, T.; Esslen, M.; Jancke, L., From emotion perception to emotion experience: Emotions evoked by pictures and classical music. International Journal of Psychophysiology 2006, 60, 34-43.

28. Seyedtabaii, S.; Seyedtabaii, L., Kalman filter based adaptive reduction of motion artifact from photoplethysmographic signal. In Proceedings of world academy of science, engineering and technology, Ardil, C., Ed. 2008; Vol. 27, pp 173-176.

29. Liu, C.Y.; Zhuang, T.; Zhao, L.N.; Chang, F.L.; Liu, C.C.; Wei, S.S.; Li, Q.Q.; Zheng, D.C., Modelling arterial pressure waveforms using gaussian functions and two-stage particle swarm optimizer. Biomed Research Int. 2014, Volume 2014, ID 923260.

30. Liu, C.Y.; Zheng, D.C.; Murray, A.; Liu, C.C., Modeling carotid and radial artery pulse pressure waveforms by curve fitting with gaussian functions. Biomed Signal Process Control 2013, 8, 449-454. 
31. Liu, C.Y.; Li, L.P.; Zhao, L.N.; Zheng, D.C.; Li, P.; Liu, C.C., A combination method of improved impulse rejection filter and template matching for identification of anomalous intervals in electrocardiographic rr sequences. J Med Biol Eng 2012, 32, 245-250.

32. Costa, T.; Galati, D.; Rognoni, E., The hurst exponent of cardiac response to positive and negative emotional film stimuli using wavelet. Auton Neurosci 2009, 151, 183-185.

33. Park, B.J.; Jang, E.H.; Kim, K.H.; Kim, S.H., Analysis of physiological responses and use of fuzzy information granulation-based neural network for recognition of three emotions. ETRI J 2015, 37, 1231-1241.

34. Sugawara, J.; Tarumi, T.; Tanaka, H., Effect of mirthful laughter on vascular function. Am J Cardiol 2010, 106, 856-859.

35. Miller, M.; Fry, W.F., The effect of mirthful laughter on the human cardiovascular system. Med Hypotheses 2009, 73, 636-639. 